\title{
Status of outreach activities at LHCb
}

\author{
Luca Pescatore* on behalf of the LHCb collaboration \\ École Polytechnique Fédérale de Lausanne \\ E-mail: luca.pescatore@cern.ch
}

The LHCb collaboration is committed to bring particle physics to a wider audience. LHCb is visible on the web with the public page news, social networks and the online event display. Masterclasses activities for school boys and girls cover the whole planet. The surface exhibition above the LHCb proton-proton collision point is being constantly developed. Laser scan of LHCb detector is performed to archive its $3 \mathrm{D}$ images as an important heritage for humanity.

XXXIX International Conference on High Energy Physics

3-11 July 2018

Seoul, South Korea

${ }^{*}$ Speaker. 
The LHCb collaboration is committed to bring particle physics to a wider audience both to share awareness on research activities to the general public and to encourage new generations to join CERN efforts to gain a better understanding of the universe. For this purpose the collaboration is involved various outreach activities including the development of didactic material and the organisation of outreach events. The status of the main activities is described in the next sections.

\section{LHCb on the web}

The web is nowadays the major source of information for most of the public and an easy way to reach a large audience. For this reasons the LHCb experiment considers important to engage the public via the web. A public page [1] is available where news are reported about every two weeks on new scientific achievements or events at CERN and LHC. LHCb is also active on the social networks and has accounts on Facebook, Twitter and Instagram with growing numbers of followers; this is becoming a fundamental channel of communication used by all LHC experiments. Finally, LHCb offers an event display publicly accessible on the web [2], where events can be seen in real time. This is an interactive tool where users can rotate the point of view, zoom and change colour assigned to different types of particles. An example can be seen in Fig. 1.

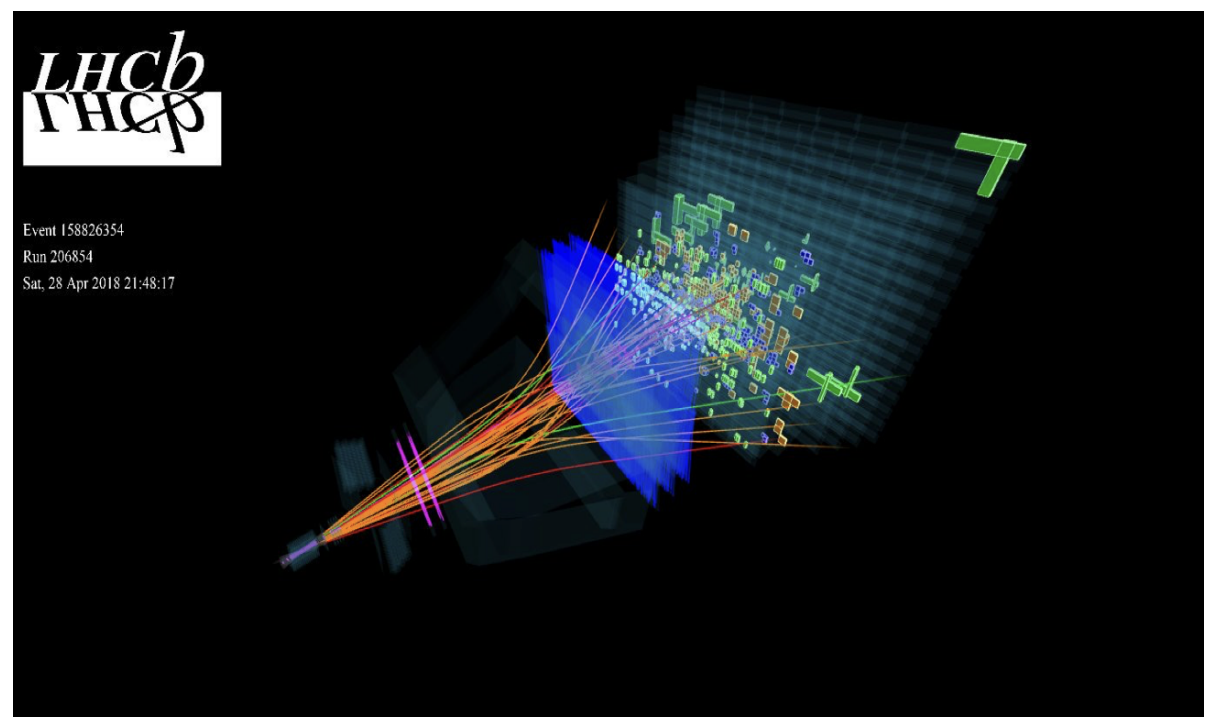

Figure 1: Publicly accessible Event Display of LHCb.

\section{Masterclasses}

The International Masterclasses [3] are events organised by the International Particle Physics Outreach Group for high school students to spread awareness about high energy physics. Each event is themed on the research of one of the four main LHC experiments. In particular LHCb took part to 10 events, connecting with 34 institutes in 13 countries in 2018. The students are presented with a research task that allows them to get a feeling of the work of a CERN researcher. The event ends with a videoconference with two CERN researchers where the results are discussed and the 
students can ask questions about physics topics but also about life as a researcher and at CERN. The project presented by LHCb in 2018 involved finding $D^{0} \rightarrow K \pi$ decays using an event display and, in a second stage, fitting the invariant mass of the obtained candidates to subtract background and measure the $D^{0}$ lifetime. The application used by the masterclass students to perform the analysis task is shown in Fig. 2. During the videoconference LHCb also offers the possibility of connecting with a scientist in the LHCb cavern, in addition to the two researchers in a room at CERN. The events had a very high success among the students and LHCb will continue to take part in the following years.

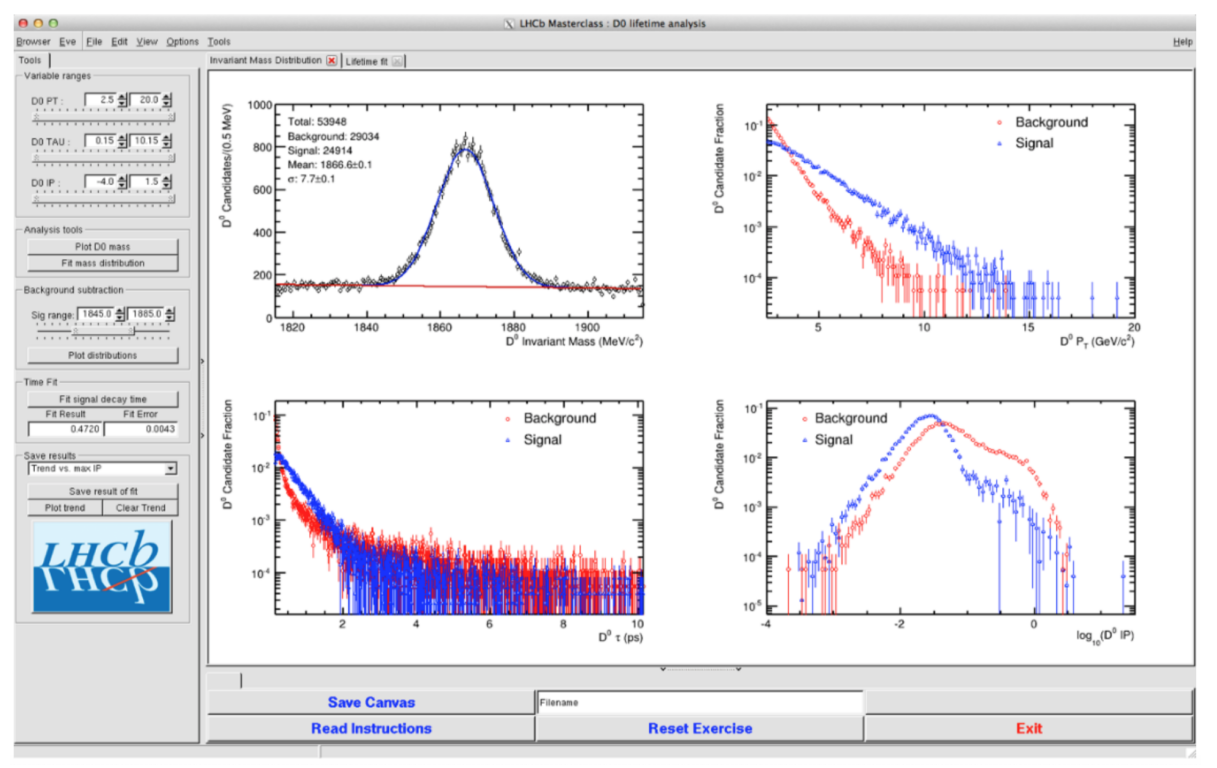

Figure 2: Application used by the masterclass students to perform the analysis task proposed by LHCb.

\section{Exhibition and didactic material}

LHCb also offers an exhibition for visitors at CERN set up in the surface buildings. This is particularly important when LHC is running and underground visits are not possible. The exhibition includes samples of various sub-detectors, calorimeters, RICH, VeLo with explanation, as well as $4 \mathrm{~m}$ long posters covering important aspects of the LHCb physics program: rare decays, $\mathrm{CP}$ violation, exotic particle searches; an example is reported in Fig. 3. To better engage the visitors, a full scale sketch of the $\mathrm{LHCb}$ detector has been drawn on the ground at the surface in the point corresponding to the actual location of the detector underground. Finally, the collaboration has also produced a didactic video covering the goals of the $\mathrm{LHCb}$ experiment and the description of the first collisions at LHC seen by an LHCb perspective [4]. A second video is being produced. Its plot tells the story of how a discovery is made taking as example the $B_{s} \rightarrow \mu \mu$ analysis, which yielded the discovery of the decay in 2014 [5]. The video starts from how the basic idea for a search is conceived and then continues with the detector development, the data taking and finally the analysis and publication process. 


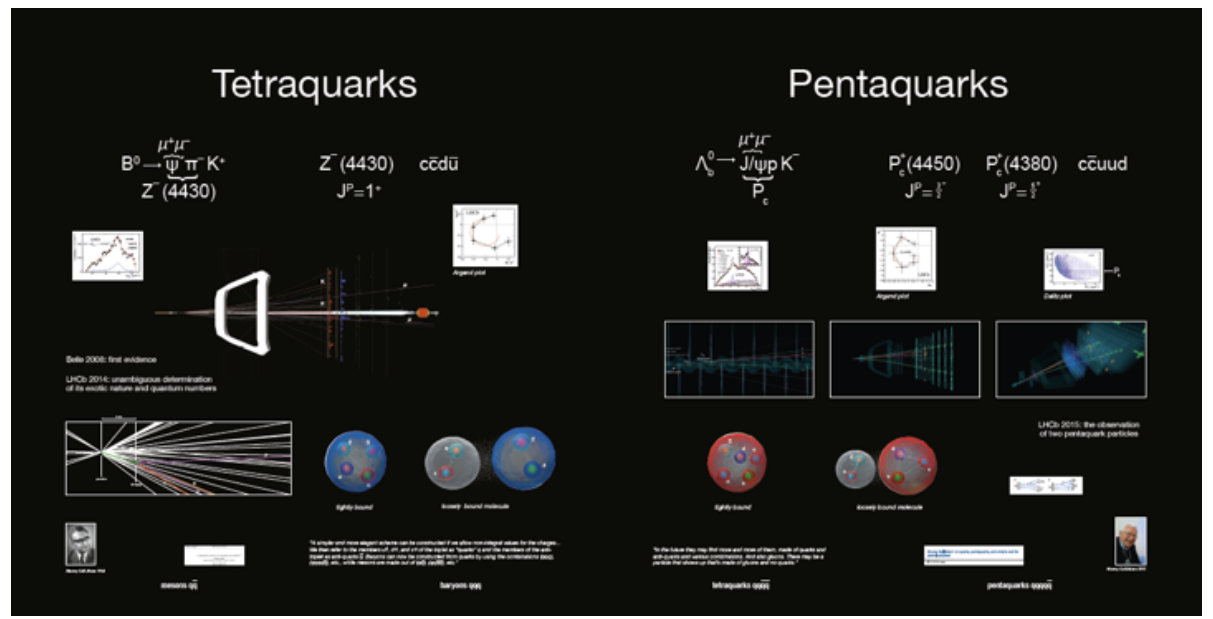

Figure 3: One of the posters in the exhibition at the LHCb site, explaining the discovery of exotic particles composed of more than three quarks.

\section{Laser scans}

High precision laser scans of the LHCb cavern were taken with a resolution of $3 \mathrm{~mm}$ at $10 \mathrm{~m}$ distance. In total 160 scans were taken including the main cavern and the neighbouring one, where the trigger computers and the Delphi experiment are located. These scans will allow to reconstruct a 3D image of the detector and its surroundings which can be used for outreach purposes including the possible creation of a virtual visit of the cavern with modern virtual reality tools.

\section{Conclusions}

The LHCb collaboration is committed to a variety of outreach activities targeting different audiences including: CERN visitors, high school students and the general public on the web. LHCb considers spreading knowledge and awareness an integrating part of research and will continue its efforts to reach an ever increasing audience in the future.

\section{References}

[1] "LHCb public page." http://lhcb-public.web.cern.ch/lhcb-public/. Accessed: 2018-11-07.

[2] "LHCb event display." https://lbevent.cern.ch/EventDisplay/index.html. Accessed: 2018-11-07.

[3] "International Masterclasses website." http://physicsmasterclasses.org. Accessed: 2018-11-07.

[4] "LHCb movie on youtube." https://www. youtube.com/watch?v=rsmBMuTFdkA. Accessed: 2018-11-07.

[5] LHCb, CMS Collaboration, V. Khachatryan et al., Observation of the rare $B_{s}^{0} \rightarrow \mu^{+} \mu^{-}$decay from the combined analysis of CMS and LHCb data, Nature 522 (2015) 68-72, [arXiv:1411.4413]. 